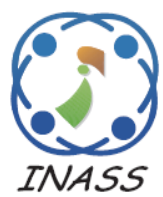

\title{
Sudden Cardiac Arrest Prediction Using Predictive Analytics
}

\author{
Anurag Bhatt ${ }^{1}$, Sanjay Kumar Dubey ${ }^{1}$, Ashutosh Kumar Bhatt ${ }^{2}$ \\ ${ }^{1}$ Amity University Uttar Pradesh, Noida, India \\ ${ }^{2}$ Birla Institute of Applied Sciences, Bhimtal,Uttarakhand, India \\ * Corresponding author's Email: anurag15bhatt@gmail.com
}

\begin{abstract}
In today's modern world, Cardiovascular Disease is one of the most lethal one. Sudden Cardiac Death is the result of this heart disease that attacks a person so instantly that it hardly gives seconds to be operated and heart freezes causing death at spot which makes it more sever and complicated for hospitals and medical services. In this paper, we have given our efforts to predict the possibility of occurring of these quick and killing attacks using decision model based predictive analytics techniques, so that we can analyze and find some patterns that are common in the happenings of Sudden Cardiac Death. Unfortunately, most hospitals and medical service organization's data are rarely used in clinical research while these datasets has such a huge potential by applying for predictive analytical approach. Classification Algorithms that comes under the decision model based in order to predict the probability of Sudden Cardiac Attack on heart disease patients. From our results, we came to know that in Hungarian database as well as in Echocardiograph database, Naïve Bayes algorithm outperformed all other algorithms and showed the maximum accuracy. In order to tackle this situation, we have proposed a framework that can be implemented for emergency situations of people with such medical history and these raw datasets are further analyzed at scratch to predict the upcoming life threatening pain that might cause death.
\end{abstract}

Keywords: SCD (Sudden Cardiac Death), J48 algorithm, Naïve Bayes algorithm, Predictive modelling, Confusion matrix.

\section{Introduction}

Predictive modeling comes under predictive analytics (a part of data mining) is evolved as a novel field to mine knowledge out of the bulky datasets that contains a huge amount of numeric, binary, statistical etc. data. Mining these data with maximum accuracy is one of the most difficult task. Nowadays, Data Mining in medical informatics and clinical research is one of the most emerging field. In this paper, we have discussed such a severe and leading cause of death i.e. Sudden Cardiac Death in heart disease patients using data mining classification algorithms, but this is not mandatory that only heart patients are vulnerable to it, it can also happen with patients with no medical history of heart disease. World Health Organization (WHO) report has recently revealed that, Cardiovascular Diseases are listed as the major cause of deaths in world, as irregular diet routine and lack of physical exercise is considered as general but effective cause behind these deaths. Large number of people die every year due to these disease than any other disease while it is estimated that about $40-50 \%$ deaths of heart disease patients are Sudden Cardiac Deaths having sudden irregular heart rhythm (arrhythmia) and only less than 1\% worldwide survives through it but, it is close to 5\% in USA and other developed countries due to increased awareness in risk factors such as smoking habits, lack of Physical exercise, irregular diet schedule etc. Sudden Cardiac Arrest as the name depicts is sudden irregularity in heart rhythm (called as arrhythmia), resulting acute chest pain may lead to death if not operated within minutes. The basic cause of having SCD and heart disease is narrowing of heart blood vessels due to excessive cholesterol, smoking, excess alcohol, poor diet and physical inactivity. This paper demonstrates the results taken from the 


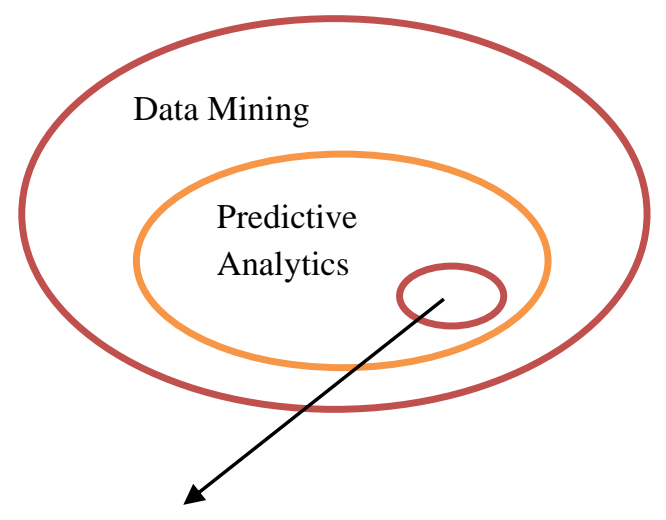

Predictive Modeling Applied

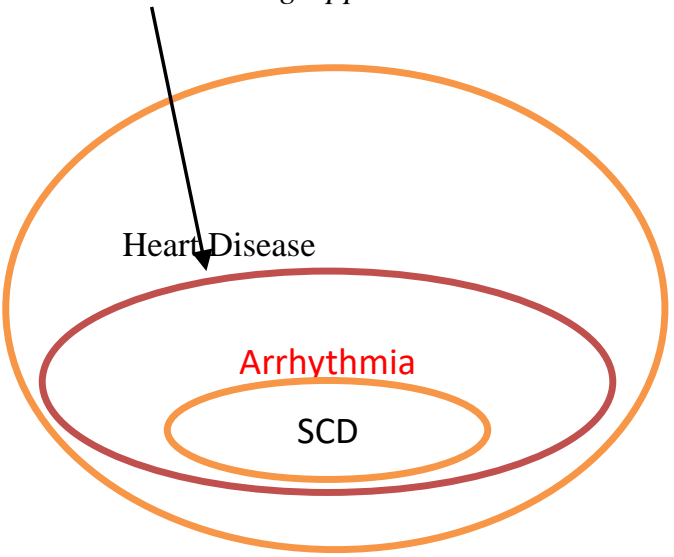

Figure.1 Relationship between Data Mining, Predictive Analytics and Predictive Modeling

experiments ran on "Weka" tool by using Hungarian heart disease dataset and heart electrical ratings based on echocardiograph reports. Various results are gathered in the form of table and their comparison to predict the occurring of SCD. The proposed method has outnumbered the existing performance of other algorithms by defining the Naïve Bayes algorithm as the most promising one with highest accuracy and precision as it is fast, highly scalable and model building algorithm.

The whole paper is organized as follows: Section 1 defines Introduction, Section 2 is Related Work and Section 3 defines Experimentation results. Section 4 is Attribute Subset Selection, Section 5 defines Analysis and prediction, Section 6 is Conclusion and Future Scope and Section 7 defines References.

\section{Related work}

The Researcher [1] has proposed a decision support system based on applying associative rule mining and classification. Associative rules are generated for classification and genetic algorithm has increased the accuracy of results. In December 2013, Abhishek Taneja [2] proposed a heart disease based prediction system using classification techniques to build a decision support system by using three different supervised algorithms i.e. Decision Tree, Bayesian Classifier and Neural Network by conducting four experiments for the study taking two situations, one containing all the 15 attributes and other containing some selected attributes, with four experiments and eight different situations and model built with J48 pruned with selected attribute scored the highest TP rate while on other hand, naïve Bayes with all attributes scored the lowest. Researchers[3] analyzed heart disease symptoms and predicted the results on applying Kmeans clustering producing cluster relevant data and then applying mafia(Maximum Frequent Item set Algorithm) that helped to recognize frequent patterns and then classification of that patterns using C4.5 algorithm. K-means based on MAFIA algorithm with ID3 and C4.5 showed accuracy of 92\%. In [4] again, $\mathrm{K}$ mean clustering is used to cluster the data and MAFIA (Maximum Frequent Item set) Algorithms used to analyze frequent patterns. Researchers [5] has introduced an approach by taking combinations of machine learning algorithms in predicting heart disease, outcome of the experiment reveals that decision tree outperforms while KNN, Neural network, Classification based on clustering algorithms didn't do well. Accuracy of decision tree and Bayesian classification further improves after applying genetic algorithm so that minimal number of attributes can be selected. Paper [6] used CART, ID3 and Decision Table algorithms with 10-fold cross validation. Prototype of system described by Researcher [7] can use two data mining algorithms i.e. Weighted Associative Classifier (WAC) and Naïve Bayes and these are further extensible, it can be used to train doctors and nurses to analyze previous record and helps doctors to predict future health complications. A review paper [8] has proposed application of data mining in the sudden cardiac death that can predict the possibility of having attack using HRV (ECG Signal derivative). Researcher[5] have used Neural Network approach to classify medical database obtained from Cleveland database and it has been seen that satisfactory results are derived from single layer and multilayer implementation of neural network. TimeFrequency analysis is done on the HRV data of patients and HRV signal is computed and Wigner Ville Transform is applied and results shows that classification is done with higher accuracy rate by Researcher [9]. Researchers in [10] and [2] has proposed a system that can predict Code Blue (an indicator for Sudden Cardiac Arrest) and can help hospital administration to predict upcoming attacks 
on the basis of analysis of previous heart patient ECG records and it also reveals that SVM (Support Vector Machine) works much better than other algorithms as it is more effective than other algorithms in terms of the data type i.e. SVM (Support Vector Machine) works better in linear separable data. In the paper [1], it has again proposed a prediction method by using associative classification algorithm using genetic approach in order to get high accuracy. This work is limited to the problem of having missing values that can produce various run time anomalies, thereby affecting the overall performance of the algorithms. Thus Researcher in paper [11] have given a review concluding with the performance on various data mining algorithms uses in heart disease prediction system. Researcher [10] has given his effort on determining an approach or method to reduce computational complexity of ECG for scalable and efficient use in large dataset.

\section{Experimentation results}

The following mathematics has done below by taking UCI machine learning repository database from which Hungarian database of heart disease is taken and Echocardiograph raw data is analyzed to develop a decision based predictive model to identify the sudden cardiac death ratio among heart disease patients. The risk of getting sudden cardiac arrest increases with the patients having medical history of heart disease that causes arrhythmia as well as rhythmic unbalance of heart. Various attributes are taken from the database that are collectively affecting the experiment results as well as it shows the efficiency or accuracy of particular algorithm applied in the data set. These two selected datasets are run with $\mathrm{J} 48$ pruned and Naïve Bayes algorithm in order to provide predictive analytics showing various results which will be analyzed later and we are going to provide confusion and performance matrix form the results generated by data mining software "Weka". We have designed and proposed a predictive decision based framework that defines the various processing stages through which medical datasets will undergo. Database contains 76 attributes and only 14 are actually used whose detailed description is listed further.

\section{Attribute subset selection}

In this paper, we have analyzed our medical data in two ways: First, by using all attributes and Second, by using selected attributes through attribute selection subset process in preprocessing tab of Weka [12]. In this way, we are basically analyzing the accuracy of prediction in order to automate decision strategies for as well as accuracy of the applied algorithm in the dataset. Keeping in view the goal of this study, we have used two different supervised algorithms i.e. Decision Tree.

For the Classification and Bayesian Classifier, four experiments are total conducted and each experiment associated with two different situations i.e. with full attributes and other selected attributes. As a whole, 8 models were developed. Now, after getting through the process of attribute selection the efficiency and accuracy of various models. Various standard metrics figures like ROC Area, TP Rate, FMeasure, accuracy were used and the whole analytical process is done under the consideration of these values. Precision will prove the correctness of data mining algorithm for developing decision models under predictive analytics techniques, so that decision taking gets easier and automated, which is another important attribute for finding optimal algorithm for particular datasets. ROC area is showing class wise distribution and used to measure the performance of algorithms.

Attribute Description (Hungarian Database)

1. Age- Numeric value of age in years

2. Sex- Binary representation, $1=$ male, $0=$ female.

3. $\mathrm{Cp}$ - Chest pain types are:

Value 1- Typical Burning Sensation in heart

Value 2- Acute stabbing like pain

Value 3- Burning Sensation

Value 4- Acute Crushing Pain in heart

4. Restbps- resting blood pressure values (numeric)

5. Chol- Cholesterol level in patient (numeric)

6. Fbs Value- fasting blood sugar level where 1 $=$ true, $0=$ false

7. Restecg- Rested electrocardiographic report indication

Value 0: normal

Value 1: ST-T wave Abnormality

Value 2: Ventricular Abnormality

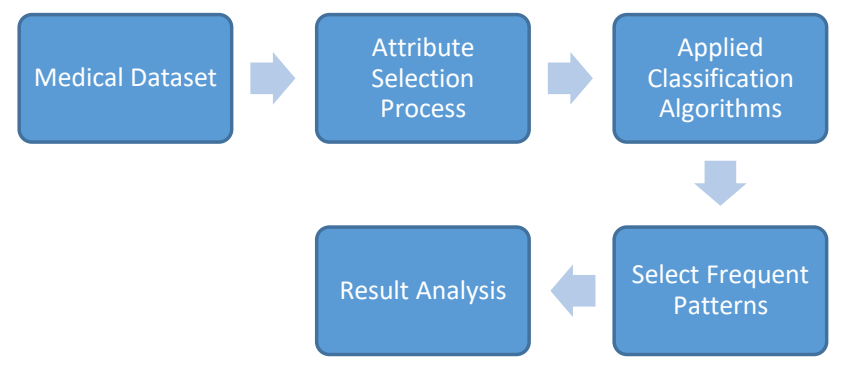

Figure.2 Proposed Framework 
8. Thalach Value- It shows maximum heart rate achieved

9. Exang- Angina caused by exercising $(1=$ yes, $0=$ no)

10. Oldpeak- ST wave depression induced by exercising relative to rest.

11. Slope- the slope of the peak exercise ST segment.

Value 1: slope in up direction.

Value 2: flat or no slope.

Value 3: downward slope.

12. Ca- number of major vessels.

13. Thal Value- It stands for Thalassemia value Value ' 3 ' = Normal.

Value ' 6 ' $=$ Fixed defect.

Value ' 7 '= Reversible defect.

14. Num: Number of Patients with heart disease. Value 0: No heart Disease

Value 1: Heart Disease Level-1

Value 2: Heart Disease Level-2

Value 3: Heart Disease Level-3

Value 4: Heart Disease Level-4

The First Experiment was conducted on Hungarian Database using J48 pruned algorithm under classification in two ways:

\section{a. Using all attributes}

b. Using selected attributes

Table 1.1 and 1.2 Confusion matrix and detailed performance of experiment 1 is listed below showing results on 14 attributes and 294 instances. Whole data is classified into 5 classes as $0,1,2,3$ and 4. " 0 " class represents "No Heart Disease" where " 1 " represents "Level-1 Heart disease", "2" represents "Level-2 Heart disease", "3" represents "Level-3 Heart Disease" and "4" represents "Level-4 heart Disease". J48 Pruned with all attributes generated a tree with a size of 158 and 156 while J48 Pruned with selected attributes generated tree of size 5 with 4 leaves. Experiment 1 achieved accuracy of " $82.3 \%$ " with all attributes and "65.64\%" with selected attributes.

\section{Experiment 1 \\ Confusion Matrix for Experiment 1}

$0=$ No Heart Disease

$1=$ Level 1 Heart Disease

$2=$ Level 2 Heart Disease

$3=$ Level 3 Heart Disease

$4=$ Level 4 Heart Disease
Table 1. J48 Pruned with all attributes

(Number of Leaves in model: 156, Tree Size: 158)

\begin{tabular}{|c|c|c|c|c|c|}
\hline $\mathbf{A}$ & $\mathbf{B}$ & $\mathbf{C}$ & $\mathbf{D}$ & $\mathbf{E}$ & Classification \\
\hline 13 & 0 & 0 & 0 & 2 & 0 \\
\hline 2 & 17 & 0 & 0 & 7 & 1 \\
\hline 3 & 3 & 13 & 0 & 9 & 2 \\
\hline 1 & 4 & 0 & 14 & 18 & 3 \\
\hline 2 & 0 & 1 & 0 & 185 & 4 \\
\hline
\end{tabular}

Table 2. J48 Pruned with Selected attributes (Number of Leaves: 4, Tree Size: 5)

\begin{tabular}{|c|c|c|c|c|c|}
\hline A & B & C & D & E & Classification \\
\hline 0 & 0 & 12 & 0 & 3 & 0 \\
\hline 0 & 1 & 15 & 0 & 10 & 1 \\
\hline 0 & 0 & 23 & 0 & 5 & 2 \\
\hline 0 & 0 & 22 & 0 & 15 & 3 \\
\hline 0 & 0 & 19 & 0 & 169 & 4 \\
\hline
\end{tabular}

Detailed Performance of Experiment 1

TP Rate: True Positive Rate

ROC Area: Receiver Operating Characteristic

F- Measure: Harmonic mean of precision and recall

Table 3. J48 pruned with all Attributes

\begin{tabular}{|c|c|c|c|c|}
\hline Accuracy & TP Rate & Precision & $\begin{array}{c}\text { F- } \\
\text { Measure }\end{array}$ & $\begin{array}{c}\text { ROC } \\
\text { Area }\end{array}$ \\
\hline $82.3 \%$ & 0.823 & 0.844 & 0.804 & 0.849 \\
\hline
\end{tabular}

Table 4. J48 pruned with selected attributes

\begin{tabular}{|c|c|c|c|c|}
\hline Accuracy & TP Rate & Precision & $\begin{array}{c}\text { F- } \\
\text { Measure }\end{array}$ & $\begin{array}{c}\text { ROC } \\
\text { Area }\end{array}$ \\
\hline $65.64 \%$ & 0.656 & 0.647 & 0.598 & 0.773 \\
\hline
\end{tabular}

Second Experiment was designed with Naïve Bayes Classification Algorithm. Table 3. Confusion matrix classified 14 attributes with 294 instances into again 5 class labels. Experiment 2 achieved accuracy of $83.33 \%$ with all attributes and accuracy of $79.59 \%$ with selected attributes.

\section{Experiment 2}

\section{Confusion Matrix for Experiment 2}

$0=$ No Heart Disease

$1=$ Level 1 Heart Disease

$2=$ Level 2 Heart Disease

$3=$ Level 3 Heart Disease

4 = Level 4 Heart Disease 
Table 5. Naïve Bayes with all attributes

\begin{tabular}{|c|c|c|c|c|c|}
\hline $\mathbf{A}$ & $\mathbf{B}$ & $\mathbf{C}$ & $\mathbf{D}$ & $\mathbf{E}$ & Classification \\
\hline 174 & 7 & 1 & 5 & 1 & 0 \\
\hline 12 & 22 & 1 & 2 & 0 & 1 \\
\hline 7 & 2 & 16 & 1 & 0 & 2 \\
\hline 5 & 1 & 0 & 22 & 0 & 3 \\
\hline 2 & 0 & 0 & 2 & 11 & 4 \\
\hline
\end{tabular}

Table 6. Naïve Bayes with selected attributes

\begin{tabular}{|c|c|c|c|c|c|}
\hline $\mathbf{A}$ & $\mathbf{B}$ & $\mathbf{C}$ & $\mathbf{D}$ & $\mathbf{E}$ & Classification \\
\hline 176 & 2 & 0 & 9 & 1 & 0 \\
\hline 15 & 19 & 2 & 1 & 0 & 1 \\
\hline 8 & 1 & 16 & 1 & 0 & 2 \\
\hline 6 & 2 & 1 & 19 & 0 & 3 \\
\hline 1 & 1 & 5 & 4 & 4 & 4 \\
\hline
\end{tabular}

\section{Detailed Performance of Experiment 2}

TP Rate: True Positive Rate

ROC Area: Receiver Operating Characteristic

F- Measure: Harmonic mean of precision and recall

Table 7. Naïve Bayes with all attributes

\begin{tabular}{|c|c|c|c|c|}
\hline Accuracy & $\begin{array}{c}\text { TP } \\
\text { Rate }\end{array}$ & Precision & $\begin{array}{c}\text { F- } \\
\text { Measure }\end{array}$ & $\begin{array}{c}\text { ROC } \\
\text { Area }\end{array}$ \\
\hline $83.33 \%$ & 0.833 & 0.834 & 0.83 & 0.954 \\
\hline
\end{tabular}

Table 8. Naïve Bayes with selected attributes

\begin{tabular}{|c|c|c|c|c|}
\hline Accuracy & $\begin{array}{c}\text { TP } \\
\text { Rate }\end{array}$ & Precision & $\begin{array}{c}\text { F- } \\
\text { Measure }\end{array}$ & $\begin{array}{c}\text { ROC } \\
\text { Area }\end{array}$ \\
\hline $79.59 \%$ & 0.796 & 0.795 & 0.784 & 0.93 \\
\hline
\end{tabular}

Time taken to build the model in Hungarian Database is 0 seconds for experiment 1 and experiment 2.

Attribute Description (Echocardiogram database)

Survival Period(in months)- Number of patients has survived of attack

Still-alive value- Binary Value Representation, $0=$ dead within a period, $1=$ Still-alive.

Age during heart attack- Age of Patient during heart attack

Pericardial effusion liquid- Denoted by Binary Value, It is a liquid present around the heart muscles where $0=$ no fluid, $1=$ fluid.

Fractional Contraction- Value showing Abnormality in heart muscle contraction

Epss Value- used to measure the degree of contraction
Lvdd Value- Stands for "Left ventricular end diastolic value" and it is diastolic dimension of left ventricle.

Wall-motion value

Wall-motion-index value

Mult value

Alive at 1 year: "0" indicates patient death within 1 year by SCD and "1" indicates patient survived for 1 year.

Experiment 3 was conducted on Echocardiogram Dataset (ignoring name and group field) with $\mathrm{J} 48$ pruned and Naïve Bayes algorithm. In Table 5, confusion matrix classified data in 2 classes " 0 " and " 1 ". " 0 " indicated patient was dead within 1 year by SCD (Sudden Cardiac Death) and "1" indicated patient was alive at 1 year. 0.02 second were taken to build model with size of tree is 3 and with 2 leaves. J48 pruned with all attributes showed accuracy of $93.24 \%$ while with selected attributes, same accuracy was noted.

\section{Experiment 3 \\ Confusion Matrix for Experiment 3}

Time taken to build model: 0.02 seconds

$0=$ Patient was dead within 1 year by SCD

$1=$ Patient was alive at 1 year

Table 9. J48 Pruned with all attributes

(Number of Leaves in the model: 2, Tree Size: 3 )

\begin{tabular}{|c|c|c|}
\hline A & B & Classified As \\
\hline 45 & 5 & 0 \\
\hline 0 & 24 & 1 \\
\hline
\end{tabular}

Table 10. J48 Pruned with Selected attributes (Number of Leaves: 2, Tree Size: 3)

\begin{tabular}{|c|c|c|}
\hline A & B & Classified As \\
\hline 45 & 5 & 0 \\
\hline 0 & 24 & 1 \\
\hline
\end{tabular}

\section{Detailed Performance of Experiment 3}

TP Rate: True Positive Rate

ROC Area: Receiver Operating Characteristic

F- Measure: Harmonic mean of precision and recall

Table 11. J48 pruned with all Attributes

\begin{tabular}{|c|c|c|c|c|}
\hline Accuracy & TP Rate & Precision & $\begin{array}{c}\text { F- } \\
\text { Measure }\end{array}$ & $\begin{array}{c}\text { ROC } \\
\text { Area }\end{array}$ \\
\hline $93.24 \%$ & 0.932 & 0.944 & 0.934 & 0.76 \\
\hline
\end{tabular}

Table 12. J48 Pruned with Selected attributes

\begin{tabular}{|c|c|c|c|c|}
\hline Accuracy & TP Rate & Precision & $\begin{array}{c}\text { F- } \\
\text { Measure }\end{array}$ & $\begin{array}{c}\text { ROC } \\
\text { Area }\end{array}$ \\
\hline $93.24 \%$ & 0.932 & 0.944 & 0.934 & 0.76 \\
\hline
\end{tabular}


Experiment 4 produced good results on applying Naïve Bayes algorithm to Echocardiogram database. Naïve Bayes with all attributes showed highest accuracy of $98.64 \%$ while with selected attributes, accuracy was 93.24.

\section{Experiment 4}

\section{Confusion Matrix for Experiment 4}

Table 13. Naïve Bayes using all attributes

\begin{tabular}{|c|c|c|}
\hline A & B & Classified As \\
\hline 49 & 1 & 0 \\
\hline 0 & 24 & 1 \\
\hline
\end{tabular}

Table 14. Naïve Bayes with selected attributes

\begin{tabular}{|c|c|c|}
\hline A & B & Classified As \\
\hline 45 & 5 & 0 \\
\hline 0 & 24 & 1 \\
\hline
\end{tabular}

\section{Detailed Performance of Experiment 4}

TP Rate: True Positive Rate

ROC Area: Receiver Operating Characteristic

F- Measure: Harmonic mean of precision and recall
Table 15. Naïve Bayes with all attributes

\begin{tabular}{|c|c|c|c|c|}
\hline Accuracy & TP Rate & Precision & $\begin{array}{c}\text { F- } \\
\text { Measure }\end{array}$ & $\begin{array}{c}\text { ROC } \\
\text { Area }\end{array}$ \\
\hline $98.64 \%$ & 0.986 & 0.987 & 0.987 & 0.946 \\
\hline
\end{tabular}

Table 16. Naïve Bayes with selected attributes

\begin{tabular}{|c|c|c|c|c|}
\hline Accuracy & TP Rate & Precision & $\begin{array}{c}\text { F- } \\
\text { Measure }\end{array}$ & $\begin{array}{c}\text { ROC } \\
\text { Area }\end{array}$ \\
\hline $93.24 \%$ & 0.932 & 0.944 & 0.934 & 0.877 \\
\hline
\end{tabular}

Time taken to build the model is 0 seconds except experiment 3 on $\mathrm{J} 48$ pruned with all attributes, here it is 0.02 seconds.

Here is summarized performance of various models on both the dataset is stored that will help in evaluating and analyzing the results of the experiment in more detailed way predicting the Sudden Cardiac Death in Heart Disease patients and discussing the role of selected attributes on calculating results

\section{Summarized performance of various models}

Table 17. Summarized Performance of Hungarian Data

\begin{tabular}{|l|c|c|c|c|c|}
\hline Model & Accuracy & TP Rate & Precision & F-Measure & Roc-Area \\
\hline J48 algorithm with all attributes & $82.3 \%$ & 0.823 & 0.844 & 0.804 & 0.849 \\
\hline J48 algorithm pruned with selected attributes & $65.64 \%$ & 0.656 & 0.647 & 0.598 & 0.733 \\
\hline Naïve Bayes algorithm with all attributes & $83.33 \%$ & 0.833 & 0.834 & 0.83 & 0.954 \\
\hline $\begin{array}{l}\text { Naïve Bayes algorithm pruned with selected } \\
\text { attributes }\end{array}$ & $79.59 \%$ & 0.796 & 0.795 & 0.784 & 0.93 \\
\hline
\end{tabular}

Table 18. Summarized Performance of Echocardiogram Data

\begin{tabular}{|l|c|c|c|c|c|}
\hline Model & Accuracy & TP Rate & Precision & F-Measure & ROC Area \\
\hline J48 algorithm with all attributes & $93.24 \%$ & 0.932 & 0.944 & 0.934 & 0.76 \\
\hline J48 algorithm pruned with selected attributes & $93.24 \%$ & 0.932 & 0.944 & 0.934 & 0.76 \\
\hline Naïve Bayes algorithm with all attributes & $98.64 \%$ & 0.986 & 0.987 & 0.987 & 0.946 \\
\hline $\begin{array}{l}\text { Naïve Bayes algorithm pruned with selected } \\
\text { attributes }\end{array}$ & $93.24 \%$ & 0.932 & 0.944 & 0.934 & 0.877 \\
\hline
\end{tabular}

As presented above in the Hungarian Database Summarized performance Table, almost every algorithm performed well. In Hungarian Database, Naïve Bayes with all attributes outperformed all other algorithms with an accuracy of $83.33 \%$, while Naïve Bayes with selected attributes achieved $79.59 \%$ accuracy, J48 pruned with all attributes showed $82.3 \%$ accuracy and J48 pruned with selected attributed showed poor performance with $65.64 \%$ accuracy. Echocardiograph database on applying J48 pruned and Naïve Bayes algorithm showed tremendous results with highest accuracy of $98.64 \%$ in this study. Naïve Bayes with all attributes did great with $98.64 \%$ and Naïve Bayes with selected attributes had 93.24\%. J48 pruned with all attributes and selected attributes achieved same accuracy of $93.24 \%$.

\section{Analysis and prediction}

In this Paper, our experiments using Weka produced eye opening results to us, defining some hidden patterns, facts and analysis that will improve the decision strategy, by providing effective decision based models. Through this study we can focus our analysis and prediction in two domains:

a. Accuracy of the classification algorithm: If we talk about the efficiency of algorithms applied on 
our data set, Naïve Bayes algorithm has really done very well among others on both the datasets. Naïve Bayes achieved higher accuracy on both the datasets excluding missing values of datasets. J48 pruned achieved maximum accuracy of $93.24 \%$ and minimum of $65.64 \%$.One important thing observed here was both the algorithms scored maximum, when applied with all attributes as compared to selected attributes. This shows that attribute selection depends not only on figures or the most effective attribute but it also depends on the type of dataset and other attributes as well. In other studies, it is found that the algorithms when run with selected attributes scored maximum accuracy as compared to when run with all attributes. Therefore, it's a contradiction and it proves that attribute selection process gives different results with different datasets.

b. Sudden Cardiac Death Analysis: The experiments conducted on both Hungarian data and Echocardiogram data has produced hidden patterns in data that can help us to predict Sudden Cardiac Death. In Heart Disease Hungarian Database, we came across some figures (Table 1) showing classification of data into heart disease levels or no heart disease. This database helps to predict the heart disease problem in the patients whose some medical measurements were taken in the laboratory. Echocardiogram data set contains heart disease patient additional medical measurements having heart rate, ECG etc. Echocardiogram data finally helps us to predict that patents having heart disease have highest probability of getting Sudden Cardiac Death and our prediction is based on 1 year time. Confusion Matrix using both J48 and Naïve Bayes with selected and all attribute (Table 5) has shown the results and from that result we can see that graph of number of sudden cardiac death under 1 year is increasing as compared to no. of people alive at 1 year.

\section{Conclusion and future scope}

After having availability of such a huge research facilities, targeting development as well as deployment of decision support systems for better understanding of medical services and clinical research using various data mining models and systems, still we are somewhere stuck to identify that icebreaking point where we will define our field of data mining and machine learning in the list of our expertise. Data mining contributed in pattern finding by using analytical toolset that helps in finding patterns, while predictive analytics used in this paper will provide the answers to the next big decision based on the results. In this paper, we were trying to build a framework that'll help to generate an automated decision model for better result delivery and continue efforts of researchers of this field will definitely build such a system with maximum efficiency. In Medical Science, various factors like age, sex, lifestyle, mental status, eating habits etc. are root cause that interrupts in our biological functioning of body and slowly leads to heart disease that increases the possibility of having sudden cardiac death. Hungarian dataset clinical markers like resting ECG, cholesterol level and heart rate played an important role in depicting heart disease while echocardiogram data set on the basis of clinical markers predicted about the mortality of heart patients. As we have already seen, Hungarian database produced outstanding results when run with Naïve Bayes algorithm with highest accuracy, taken all attributes at a time while both Naïve Bayes and J48 pruned algorithms produced great results when run with echocardiograph database. Our proposed system was unable to fill certain gaps required in treatment of missing/ impartial clinical data that can be treated using various techniques like Bernoulli mixture models and expectation maximization techniques.

The future work for this paper holds the great potential for researchers to analyze various data mining algorithms with their variants in large amount of data. Hence, this gap further widens the opportunities for researchers to work in the proper treatment of missing/impartial values. Naïve Bayes algorithm with all attributes (through attribute selection) outperformed in both the databases. In a nutshell, we can conclude that SCD is most dangerous situation for both heart disease and no heart disease patients that depends on the medical history as well as life style factors of a human being and the process of decision making and predictions will be made more concise and precise by using predictive analytics to generate automated decision based model.

\section{References}

[1] M. A. Jabbar, P. Chandra, and B.L. Deekshatulu, "Heart Disease Prediction System using Associative Classification and Genetic Algorithm", In: Proc. of the International Conference on Emerging Trends in Electrical, Electronics and Communication technologiesICECIT, pp.183-192 2012.

[2] A. Taneja, "Heart Disease Prediction System Using Data Mining Techniques", Oriental 
Journal of Computer Science and technology, Vol. 6, No. 4, pp. 457-466, 2013.

[3] K. Pandey, P. Pandey, K.L. Jaiswal, A.K. Sen, "Data Mining Clustering Techniques in the Prediction of Heart Disease using Attribute selection Method," International Journal of Science, Engineering and Technology research, Vol. 2, No. 10, pp. 2003-2008, 2013.

[4] G. Karthiga, C. Preethi, and R.D. Devi, “ Heart Disease Analysis System using Data Mining Techniques", International Journal of Innovative research in Science, Engineering and Technology, Vol. 3, No. 3, pp. 3101-3105, 2014.

[5] K.U. Rani, "Analysis of Heart Disease Dataset using Neural Network Approach", International Journal of Data Mining \& Knowledge Management Process, Vol.1, No.5, pp. 1-8, 2011.

[6] V. Chaurasia and S. Pal, "Early Prediction of Heart disease using Data Mining Techniques", Caribbean Journal of Science and Technology, Vol. 1, pp. 208-217, 2013.

[7] N. Aditya Sundar, P.Pushpa Latha, nd M. Rama Chandra, "Performance Analysis of Classification Data Mining Techniques over Heart Disease Database", International Journal of Engineering Science and Advanced Technology, Vol.2, No.3, pp.470-478, 2012.

[8] L.Murukesan, M.Murugappan, and M. Iqbal, "Sudden Cardiac Death Prediction using ECG Signal Derivative (Heart Rate Variability): A Review", In: Proc. of the 2013 IEEE $9^{\text {th }}$ International Colloqium on Signal Processing and its Application, pp. 269-274, 2013.

[9] E. Ebrahimzadeh and M. Pooyan, "Early Detection of Sudden Cardiac Death by using Classical Linear techniques and TimeFrequency methods on Electrocardiogram signals," Journal of Biomedical science and Engineering, pp.699-706,2011.

[10] S. Somanchi, S. Adhikari, A. Lin, E. Eneva, and R. Ghani, "Early Prediction of Cardiac Arrest (Code Blue) using Electronic Medical Records", ACM, pp.2119-2126, 2015

[11] W.S. Kaur, "Review on Heart Disease Prediction System using Data Mining Techniques", International Journal on Recent and Innovation trends in computing and Communication, Vol. 2, No. 10, pp. 3003-3008, 2014.

[12] Weka, "Data Mining Machine Learning Software, [Online] Available http://www.cs.waikato.ac.nz/ml/. 